\title{
On the stable equivalence of open books in three-manifolds
}

\author{
EMMANUEL GIROUX \\ NOAH GOODMAN
}

\begin{abstract}
We show that two open books in a given closed, oriented three-manifold admit isotopic stabilizations, where the stabilization is made by successive plumbings with Hopf bands, if and only if their associated plane fields are homologous. Since this condition is automatically fulfilled in an integral homology sphere, the theorem implies a conjecture of $\mathbf{J}$ Harer, namely, that any fibered link in the three-sphere can be obtained from the unknot by a sequence of plumbings and deplumbings of Hopf bands. The proof presented here involves contact geometry in an essential way.
\end{abstract}

57M50, 57R17; 57M25, 57R52

Let $M$ be an oriented three-manifold. An open book in $M$ (also called open book decomposition of $M)$ is a pair $(K, \theta)$ consisting of:

- a proper one-dimensional submanifold $K$ in $M$;

- a fibration $\theta: M \backslash K \rightarrow \mathbf{S}^{1}=\mathbb{R} / 2 \pi \mathbb{Z}$ which, in some neighborhood $N=\mathbf{D}^{2} \times K$ of $K=\{0\} \times K$, is the normal angular coordinate.

The submanifold $K$ is called the binding of the open book while the closures of the fibers of $\theta$ are named pages. The binding and the pages are cooriented by $\theta$, and hence they are oriented since $M$ is. On the other hand, any page $F$ of an open book $(K, \theta)$ completely determines $K=\partial F$ and also (though much less evidently, as seen in the work of Cerf [1], Laudenbach and Blank [9] and Waldhausen [15]) $\theta$ up to isotopy relative to $F$.

Around 1920, as a corollary of his results on branched covers and the braiding of links, Alexander proved the existence of open books in any closed oriented three-manifold $M$. On the other hand, given an open book in $M$, many others can be constructed by the following plumbing operation. Let $F \subset M$ be a compact surface with boundary and $C \subset F$ a proper simple arc. We say that a compact surface $F^{\prime} \subset M$ is obtained from $F$ by $H^{ \pm}$-plumbing along $C$ - or, more explicitly, by plumbing a positive/negative Hopf band along $C-$ if $F^{\prime}=F \cup A^{ \pm}$where $A^{ \pm}$is an annulus in $M$ with the following properties:

- the intersection $A^{ \pm} \cap F$ is a tubular neighborhood of $C$ in $F$; 
- the core curve of $A^{ \pm}$bounds a disk in $M \backslash F$ and the linking number of the boundary components of $A^{ \pm}$is equal to \pm 1 .

According to results of J Stallings [11] (see Section 1), if $F$ is a page of an open book $(K, \theta)$ in $M$ then any surface $F^{\prime}$ obtained from $F$ by $H^{ \pm}$-plumbing is also a page of an open book $\left(K^{\prime}, \theta^{\prime}\right)$ in $M$. We will say that the open book $\left(K^{\prime}, \theta^{\prime}\right)$ itself is obtained from $(K, \theta)$ by $H^{ \pm}$-plumbing. A stabilization of an open book $(K, \theta)$ is an open book $\left(K^{\prime}, \theta^{\prime}\right)$ that can be obtained from $(K, \theta)$ by finitely many successive $H^{ \pm}$-plumbings.

If $M$ is closed, any open book $(K, \theta)$ in $M$ provides a Heegaard spitting of $M$ : given two antipodal values in $\mathbf{S}^{1}$, the two corresponding pages form a (smooth) closed surface dividing $M$ into handlebodies. In other words, open books may be regarded as special Heegaard splittings, namely, those for which the splitting surface contains a graph whose inclusion in the handlebody on each side is a homotopy equivalence (a regular neighborhood of this graph is then a page of the open book, as well as the closure of its complement in the surface). A well-known theorem by K Reidemeister and J Singer shows that any two Heegaard splittings of a given closed oriented three-manifold admit isotopic stabilizations, where the stabilization here is made by successive attachings of trivially embedded one-handles. Furthermore, if an open book $\left(K^{\prime}, \theta^{\prime}\right)$ is a stabilization of another one $(K, \theta)$, the associated Heegard splitting is a stabilization of the one associated with $(K, \theta)$. It is therefore natural to ask whether any two open books in a given closed oriented three-manifold have isotopic stabilizations. To answer this question, we need one more ingredient.

To any open book $(K, \theta)$ in $M$, we can associate an oriented plane field $\xi$ on $M$ in the following way: outside some product neighborhood $N=\mathbf{D}^{2} \times K$ of $K$ in which $\theta$ is the normal angular coordinate, $\xi$ is just the plane field tangent to the pages, that is, the kernel of $D \theta$; inside $N$, using oriented cylindrical coordinates $(r, \theta, z)$ with $z \in \mathbf{S}^{1} \sqcup \cdots \sqcup \mathbf{S}^{1}$ parameterizing $K$, we define $\xi$ by the form $f(r) d z+r^{2} d \theta$ where $f:[0,1] \rightarrow[0,1]$ is positive near 0 and zero near 1 . Clearly, the homotopy class of this oriented plane field does not depend on the choice of $N$ and $r, z, f$ : this is an invariant of the open book that L Rudolph considered as an "enhanced Milnor number" (L Rudolph was actually interested in the case $M=\mathbf{S}^{3}$ where homotopy classes of plane fields are parameterized by $\mathbb{Z}$ through the Hopf invariant: see the proof of Corollary 5). The main result of this paper is the following:

Theorem 1 Two open books in a closed oriented three-manifold admit isotopic stabilizations if and only if their associated oriented plane fields are homologous. 
An oriented hyperplane field on a closed oriented $n$-manifold $M$ is a section of the sphere cotangent bundle $S T^{*} M$. Two hyperplane fields are homologous if they define equal homology classes in $H_{n}\left(S T^{*} M ; \mathbb{Z}\right)$, or equivalently, if the curve in $M$ consisting of points where they coincide with opposite orientations is nullhomologous (see Section 2).

If $M$ is an integral homology three-sphere, any two plane fields on $M$ are homologous. Moreover, an open book in $M$ is completely determined up to isotopy by its oriented binding (for this, which again follows from the results of Cerf [1], Laudenbach and Blank [9] and Waldhausen [15], it actually suffices that $M$ be a rational homology three-sphere). Now recall that a link (namely, a closed oriented one-dimensional submanifold) in a closed three-manifold $M$ is a fibered link if it is the oriented binding of some open book in $M$. Thus, using the same terminology for fibered links as for open books, we get:

Corollary 2 Any two fibered links in an integral homology three-sphere admit isotopic stabilizations.

In the case of the three-sphere itself, the unknot is a fibered link, and so we get the following result conjectured by Harer [8]:

Corollary 3 Any fibered link in the three-sphere can be obtained from the unknot by finitely many plumbings and "deplumbings" of Hopf links.

These corollaries also admit specific variants for fibered knots, in which the $H^{ \pm}$ plumbings leading to the common stabilization can be performed two by two so as to give a fibered knot at each stage; in other words, the plumbing of Hopf links is replaced by the plumbing of positive trefoil knots and figure-eight knots (see Section 1 for definitions):

Corollary 4 Any two fibered knots in an integral homology three-sphere admit isotopic stabilizations obtained from each knot by finitely many plumbings of positive trefoil knots and figure-eight knots.

Following Neumann and Rudolph [10], we can rephrase the above results concerning the three-sphere in terms of the Grothendieck groups of fibered links and of fibered knots (see Section 1 for the definition):

Corollary 5 The Grothendieck group of fibered links in the three-sphere is the free Abelian group of rank two generated by the positive and the negative Hopf links, $\mathrm{H}^{+}$ 
and $H^{-}$. Similarly, the Grothendieck group of fibered knots in the sphere is the free Abelian group of rank two generated by the positive trefoil knot and the figure-eight knot.

Let's now say a couple of words about the proof of Theorem 1. The "only if" part is quite easy: an $H^{ \pm}$-plumbing yields an open book that coincides with the original one in the complement of a ball, and so the homology class of the associated plane field does not change. To prove the "if" part, we use an invariant of open books more subtle than a homotopy class of plane fields, namely, an isotopy class of contact structures. The main feature of this refined invariant is that, according to a previous article by the first author [6], it determines the open book up to positive stabilization, that is, stabilization involving only $\mathrm{H}^{+}$-plumbings (see Theorem 14). To conclude, we combine this result with a few observations on the effect of $\mathrm{H}^{-}$-plumbing on our open book invariants and the classification by Eliashberg [3] of the so-called overtwisted contact structures.

Acknowledgments The authors thank the American Institute of Mathematics for its support during the Fall of 2000. In September 2000, the first author gave a series of lectures at Stanford University on the correspondence between contact structures and open books. The second author was attending these lectures, and both independently obtained the results of this paper a few weeks later.

The first author also thanks the Centre National de la Recherche Scientifique for funding his research, and he is very grateful to Alexis Marin for his thoughtful comments on Part B of the text.

\section{Plumbing}

Let's first discuss plumbing more carefully, in slightly greater generality (see the articles by Gabai [4; 5], Harer [8] and Stallings [11] for further information). For $j \in\{1,2\}$, let $F_{j}$ be a compact oriented surface in a closed oriented three-manifold $M_{j}$ and let $C_{j} \subset F_{j}$ be a proper simple arc. We say that a compact surface $F$ in the connected sum $M=M_{1} \# M_{2}$ is obtained by plumbing $F_{1}$ and $F_{2}$ along $C_{1}$ and $C_{2}$ if $F=F_{1} \cup F_{2}$ and $F_{1} \cap F_{2}$ is a square with median segments $C_{1}$ and $C_{2}$. Thus, the $H^{ \pm}$-plumbing defined in the introduction is nothing but a plumbing with a positive/negative Hopf band in $\mathbf{S}^{3}$ (that is, an embedded annulus whose boundary components have linking number \pm 1 ) where the arc used in this band connects the two boundary components.

To see that a surface obtained by plumbing pages of two open books is still a page of an open book, we start with a simple observation. Consider in $\mathbb{R}^{3}$ the (piece of) open 
book $(\hat{K}, \hat{\theta})$ whose binding $\widehat{K}$ consists of the two lines $\{x= \pm 1, y=0\}$ and whose map $\hat{\theta}: \mathbb{R}^{3} \backslash \widehat{K} \rightarrow \mathbf{S}^{1}$ is given by

$$
\hat{\theta}(x, y, z)=\arg \left(\frac{1+x+i y}{1-x-i y}\right)=\arg \left(1-x^{2}-y^{2}+2 i y\right) .
$$

(Each page of this open book is half of a vertical cylinder containing $\widehat{K}$.)

Let $\widehat{B}$ denote the domain $\left\{x^{2}+2 y^{2}+z^{2} \leq 2\right\}$ and $\widehat{S}$ the ellipsoid $\partial \widehat{B}$. The map

$$
\hat{\rho}: \hat{S} \longrightarrow \widehat{S}, \quad(x, y, z) \longmapsto(z,-y,-x),
$$

is an orientation-reversing self-diffeomorphism of order four which permutes the four points of $\hat{S} \cap \widehat{K}$ cyclically. Moreover, for $(x, y, z) \in \hat{S}$, the identity $y^{2}+z^{2}-1=$ $1-x^{2}-y^{2}$ implies that

$$
\hat{\theta} \circ \hat{\rho}(x, y, z)=\arg \left(1-z^{2}-y^{2}-2 i y\right)=\arg \left(x^{2}+y^{2}-1-2 i y\right)=\hat{\theta}(x, y, z)+\pi .
$$

Now let $\left(K_{1}, \theta_{1}\right)$ and $\left(K_{2}, \theta_{2}\right)$ be open books in closed oriented three-manifolds $M_{1}$ and $M_{2}$, respectively, and, for $j \in\{1,2\}$, let $C_{j}$ be a proper simple arc in the page $\theta_{j}^{-1}(0) \cup K_{j}$. Each $C_{j}$ has a (big) neighborhood $W_{j}$ with an orientation-preserving diffeomorphism $\phi_{j}: W_{j} \rightarrow \mathbb{R}^{3}$ taking $\left(K_{j} \cap W_{j}, \theta_{j} \mid W_{j}\right)$ to $(\hat{K}, \hat{\theta})$ and $C_{j}$ to the unit segment $\widehat{C}$ of the $x$-axis. Hence the map

$$
\rho=\phi_{2}^{-1} \circ \hat{\rho} \circ \phi_{1}: S_{1}=\phi_{1}^{-1}(\widehat{S}) \longrightarrow S_{2}=\phi_{2}^{-1}(\widehat{S})
$$

is an orientation-reversing diffeomorphism and satisfies $\theta_{2} \circ \rho=\theta_{1}+\pi$. Therefore, the connected sum

$$
M=M_{1} \# M_{2}=\left(M_{1} \backslash \operatorname{Int} B_{1}\right) \underset{\rho}{\cup}\left(M_{2} \backslash \operatorname{Int} B_{2}\right), \quad B_{j}=\phi_{j}^{-1}(\widehat{B}),
$$

is naturally equipped with an open book $(K, \theta)$ whose binding is the union $\left(K_{1}-\right.$ Int $\left.B_{1}\right) \cup\left(K_{2} \backslash\right.$ Int $\left.B_{2}\right)$ and whose fibration $\theta$ is equal to $\theta_{j}+(-1)^{j+1} \pi / 2$ on $M_{j} \backslash$ Int $B_{j}, j \in\{1,2\}$. Moreover, the 0 -page of $(K, \theta)$ is (easily seen to be) obtained by plumbing the $-\pi / 2$-page of $\left(K_{1}, \theta_{1}\right)$ and the $\pi / 2$-page of $\left(K_{2}, \theta_{2}\right)$ along the $\operatorname{arcs} C_{1}^{\prime}$ and $C_{2}^{\prime}$ defined by

$$
\begin{aligned}
\phi_{1}\left(C_{1}^{\prime}\right) & =\left\{x^{2}+y^{2}=1, y \leq 0, z=0\right\}, \\
\text { and } \quad \phi_{2}\left(C_{2}^{\prime}\right) & =\left\{x^{2}+y^{2}=1, y \geq 0, z=0\right\} .
\end{aligned}
$$

The open book $(K, \theta)$ is said to be obtained by plumbing $\left(K_{1}, \theta_{1}\right)$ and $\left(K_{2}, \theta_{2}\right)$ along $C_{1}$ and $C_{2}$. 
In the last section of this paper (see the proof of Lemma 16), we will call plumbing ball for an open book $(K, \theta)$ in $M$ any ball $B \subset M$ such that there exists a diffeomorphism $B \rightarrow \widehat{B}$ which takes $\left(K \cap B, \theta \mid{ }_{B}\right)$ to $\left(\widehat{K} \cap \widehat{B},\left.\widehat{\theta}\right|_{\widehat{B}}\right)$.

Example 6 Consider $\mathbf{S}^{3}$ as the unit sphere in $\mathbb{C}^{2}$ :

$$
\mathbf{S}^{3}=\left\{\left(r_{1} e^{i \theta_{1}}, r_{2} e^{i \theta_{2}}\right) \mid r_{1}^{2}+r_{2}^{2}=1, \theta_{1}, \theta_{2} \in \mathbb{R} / 2 \pi \mathbb{Z}\right\} .
$$

The (unoriented) Hopf link $H=\left\{r_{1} r_{2}=0\right\} \subset \mathbf{S}^{3}$ is the binding of two open books given by the maps

$$
\theta^{ \pm}: \mathbf{S}^{3} \backslash H \longrightarrow \mathbf{S}^{1}, \quad\left(r_{1} e^{i \theta_{1}}, r_{2} e^{i \theta_{2}}\right) \longmapsto \theta_{1} \pm \theta_{2} .
$$

These two maps orient $H$ in different ways, and we will denote by $H^{ \pm}$the Hopf link equipped with the orientation induced by $\theta^{ \pm}$(in particular, the linking number of the components of $H^{ \pm}$is \pm 1 ). On the other hand, the unknot $U=\left\{r_{1}=0\right\}$ is the binding of an open book whose fibration is the map $\theta_{1}$.

Now let $(K, \theta)$ be an open book in a closed oriented three-manifold $M$ and $C$ a proper simple arc in one of its pages. The open book in $M \# \mathbf{S}^{3}=M$ obtained by plumbing $(K, \theta)$ with $\left(H^{ \pm}, \theta^{ \pm}\right)$along $C$ and an arc connecting the two components of the Hopf link is what we called earlier the open book obtained by $H^{ \pm}$-plumbing along $C$. On the other hand, plumbing $(K, \theta)$ with $\left(U, \theta_{1}\right)$ - along any arcs — always yield an open book isotopic to $(K, \theta)$.

By plumbing together $\left(H^{+}, \theta^{+}\right)$and $\left(H^{\varepsilon}, \theta^{\varepsilon}\right), \varepsilon \in\{+,-\}$, along arcs joining the two boundary components, we obtain an open book in $\mathbf{S}^{3}$ whose binding is the positive trefoil knot $T^{+}$if $\varepsilon=+$ and the figure-eight knot $E$ if $\varepsilon=-$. The plumbing operations with the open books so obtained will be called $T^{+}{ }_{-}$plumbing and $E_{-}$ plumbing, respectively, provided the arc used in the punctured-torus Seifert surface of $T^{+}$or $E$ is non-separating.

The plumbing operation allows us to define a Grothendieck group for fibered links in the three-sphere (see the article [10] by Neumann and Rudolph). It is the group generated by all (isotopy classes of) fibered links in $\mathbf{S}^{3}$ in which we impose the relation $[K]=\left[K^{\prime}\right]+\left[K^{\prime \prime}\right]$ for any triple of fibered links $\left(K, K^{\prime}, K^{\prime \prime}\right)$ such that $K$ is obtained by plumbing $K^{\prime}$ and $K^{\prime \prime}$ in some way. Clearly, this group is Abelian - because plumbing is a commutative operation - and its identity element is the unknot $U$. The Grothendieck group of fibered knots in the sphere is defined similarly from the set of all (isotopy classes of) fibered knots in $\mathbf{S}^{3}$. 


\section{Hyperplane fields}

Let $M$ be a connected, oriented $n$-manifold with zero Euler characteristic. We denote by $\mathcal{P} \mathcal{F}(M)$ the (non-empty) space of (co) oriented hyperplane fields on $M$ and by $\mathcal{P} \mathcal{F}_{c}(M)$ its subspace consisting of hyperplane fields that coincide with a fixed one (arbitrarily chosen) outside of a compact subset of Int $M$, that is, near the boundary and at infinity - so $\mathcal{P} \mathcal{F}_{c}(M)=\mathcal{P} \mathcal{F}(M)$ if $M$ is closed. This section is a digression in which we investigate the structure of the set $\pi_{0} \mathcal{P} \mathcal{F}_{c}(M)$ of connected components of $\mathcal{P} \mathcal{F}_{c}(M)$ - or homotopy classes of hyperplane fields. There is nothing new in our discussion, whose key ideas are due to H Hopf and L Pontryagin, but we include it since what we need is elementary and apparently not so well known (see, however, the articles by Dufraine [2], Gompf [7] and Turaev [14]).

Let $\xi, \eta \in \mathcal{P} \mathcal{F}_{c}(M)$ be hyperplane fields and let $\alpha, \beta$ denote respective defining one-forms which coincide near the boundary and at infinity. The first obstruction to the existence of a path joining $\xi$ to $\eta$ inside $\mathcal{P F}_{c}(M)$ is a homology class $c(\xi, \eta)$ in $H_{1}(M ; \mathbb{Z})=H_{1}([0,1] \times M ; \mathbb{Z})$, namely the class of the zero set of a generic homotopy between $\alpha$ and $\beta$ with compact support in $[0,1] \times \operatorname{Int} M$. Clearly, for any $\xi, \eta, \zeta \in \mathcal{P F}_{c}(M)$, the following cocycle relations hold:

$$
\begin{aligned}
c(\xi, \xi) & =0 \\
c(\xi, \eta)+c(\eta, \xi) & =0 \\
c(\xi, \eta)+c(\eta, \zeta)+c(\zeta, \xi) & =0
\end{aligned}
$$

On the other hand, if the linear homotopy $(1-t) \alpha+t \beta, t \in[0,1]$, is generic (that is, transverse to the zero section), then the projection to $M$ of its zero set is the curve $C(\xi, \eta)$ of points where $\xi$ coincides with $-\eta$.

The obstruction class $c(\xi, \eta)$ can also be viewed differently, assuming for instance that $M$ is closed. As sections of the sphere cotangent bundle $S T^{*} M$, the hyperplane fields $\xi$ and $\eta$ determine homology classes $[\xi],[\eta] \in H_{n}\left(S T^{*} M ; \mathbb{Z}\right)$. Then consider the long homology exact sequence

$$
\cdots \rightarrow H_{n+1}\left(B T^{*} M, S T^{*} M ; \mathbb{Z}\right) \rightarrow H_{n}\left(S T^{*} M ; \mathbb{Z}\right) \rightarrow H_{n}\left(B T^{*} M ; \mathbb{Z}\right) \rightarrow \cdots,
$$

where $B T^{*} M$ denotes the ball cotangent bundle. The classes $[\xi],[\eta]$ have the same image in $H_{n}\left(B T^{*} M ; \mathbb{Z}\right)$, and so the difference $[\xi]-[\eta]$ is the image of a class $\widetilde{c}(\xi, \eta) \in$ $H_{n+1}\left(B T^{*} M, S T^{*} M ; \mathbb{Z}\right)$ and $c(\xi, \eta) \in H_{1}(M ; \mathbb{Z})$ is just the intersection of $\widetilde{c}(\xi, \eta)$ with the class of the zero section in $H_{n}\left(B T^{*} M\right.$; $\left.\mathbb{Z}\right)$. Thus, $c(\xi, \eta)=0$ if and only if $[\xi]=[\eta]$. 
We now need to distinguish homotopy classes of homologous hyperplane fields. Our tool here is an action of the group $\pi_{n} S^{n-1}$ on the set $\pi_{0} \mathcal{P} \mathcal{F}(M)$. Take a hyperplane field $\xi \in \mathcal{P} \mathcal{F}(M)$, and choose an orientation-preserving embedding $\phi: \mathbf{D}^{n} \rightarrow M$ with $B=\phi\left(\mathbf{D}^{n}\right)$. The derivative $d \phi$ is homotopic to a trivialization $\widetilde{d \phi}: \mathbf{D}^{n} \times\left.\mathbb{R}^{n} \rightarrow T M\right|_{B}$ (covering $\phi$ ) in which the hyperplane field $\left.\xi\right|_{B}$ is the kernel of the one-form $\widetilde{d \phi}_{*} d x_{n}$. Any map $g: \mathbf{D}^{n} \rightarrow \mathbf{S}^{n-1}$ that is constant equal to $(0, \ldots, 0,1)$ near $\partial \mathbf{D}^{n}$ can then be used to construct a new hyperplane field $\eta=(g \cdot \xi)_{\widetilde{d \phi}}$ on $M$ : set $\eta=\xi$ out of $B$ and, regarding $\mathbf{S}^{n-1}$ as the unit sphere of the dual space $\left(\mathbb{R}^{n}\right)^{*}$, define $\eta(p)$ for $p \in B$ to be the kernel of the linear form $\widetilde{d \phi}_{*} g(p)$.

This construction induces a group action of $\pi_{n} \mathbf{S}^{n-1}$ on $\pi_{0} \mathcal{P} \mathcal{F}(M)$ and $\pi_{0} \mathcal{P} \mathcal{F}_{c}(M)$. First observe that, since $M$ is connected as well as the implied spaces of embeddings and trivializations, the homotopy class of the hyperplane field $(g \cdot \xi) \widetilde{d \phi}$ is unsensitive to the choice of $\phi, \widetilde{d \phi}$ and depends only on the homotopy classes of $\xi$ and $g$. Next, the constant map $g=(0, \ldots, 0,1)$ acts trivially. Finally, to check the composition rule, denote by $\widetilde{d \phi}_{ \pm}$the restriction of $\widetilde{d \phi}$ to $\mathbf{D}_{ \pm}^{n} \times \mathbb{R}^{n}$ where

$$
\mathbf{D}_{ \pm}^{n}=\left\{\left(x-1, \ldots, x_{n}\right) \in \mathbf{D}^{n} \mid \pm x_{n} \geq 0\right\} .
$$

If $g: \mathbf{D}_{-}^{n} \rightarrow \mathbf{S}^{n-1}$ and $h: \mathbf{D}_{+}^{n} \rightarrow \mathbf{S}^{n-1}$ are maps equal to $(0, \ldots, 0,1)$ near the boundary and if $g h$ refers to the resulting map $\mathbf{D}^{n} \rightarrow \mathbf{S}^{n-1}$ - whose homotopy class is the product of the homotopy classes of $g$ and $h-$, then

$$
(g h \cdot \xi)_{\widetilde{d \phi}}=\left(g \cdot(h \cdot \xi)_{\widetilde{d \phi}}\right) \widetilde{d \phi}_{-} .
$$

This action and the obstruction cocycle determine the homotopy classification of hyperplane fields on $M$ :

Proposition 7 Let $M$ be a connected, oriented manifold of dimension $n \geq 2$ with zero Euler characteristic. The continuous cocycle

$$
c: \mathcal{P} \mathcal{F}_{c}(M) \times \mathcal{P} \mathcal{F}_{c}(M) \longrightarrow H_{1}(M ; \mathbb{Z})
$$

is surjective and two hyperplane fields $\xi, \eta$ satisfy $c(\xi, \eta)=0$ if and only if their homotopy classes are in the same orbit of $\pi_{n} \mathbf{S}^{n-1}$. Furthermore, the stabilizer in $\pi_{n} \mathbf{S}^{n-1}$ of the homotopy class of any hyperplane field $\xi$ is trivial if $n \geq 4$ and, for $n=3$, is the image of the homomorphism $H_{2}(M ; \mathbb{Z}) \rightarrow \pi_{1} \mathrm{SO}_{2}=\pi_{3} \mathbf{S}^{2}$ defined by the Euler class of $\xi$.

Strictly speaking, as an obstruction class, the Euler class of $\xi$ belongs to $H^{2}\left(M, \pi_{1} \mathbf{S}^{1}\right)$, and so the implied homomorphism is rather given by the $\pi_{1} \mathrm{SO}_{2}$-valued lift of the 
second Stiefel-Whitney class of $\xi$. On the other hand, $\pi_{1} \mathrm{SO}_{2}$ is identified with $\pi_{3} \mathbf{S}^{2}$ via the Pontryagin isomorphism (see Example 9 below).

The proof of this proposition is actually more instructive than its statement. We first recall a simple fact: for any finite dimensional vector space $E$, the tangent space to the Grassmann manifold $\mathbf{G}_{k}(E)$ at each point $\tau$ (a vector subspace of dimension $k$ in $E$ ) can be canonically identified with $\tau^{*}=\operatorname{Hom}(\tau, E / \tau)$. With this in mind, the key observation is the following:

Lemma 8 Let $M$ be a connected, oriented manifold of dimension $n \geq 2$ with zero Euler characteristic. Given a hyperplane field $\xi \in \mathcal{P} \mathcal{F}_{c}(M)$, there is a natural oneto-one correspondence between $\pi_{0} \mathcal{P} \mathcal{F}_{c}(M)$ and the set $\Omega_{1}\left(M ; \xi^{*}\right)$ of cobordism classes of $\xi^{*}$-framed curves in $M$.

A $\xi^{*}$-framed curve is a pair $(C, \gamma)$ consisting of a closed one-dimensional submanifold $C$ in $M$ and a bundle equivalence (that is, a bundle isomorphism over the identity) $\gamma:\left.v C \rightarrow \xi^{*}\right|_{C}$, where $v C$ denotes the normal bundle of $C$ and $\xi^{*}$ the bundle $\operatorname{Hom}(\xi, T M / \xi)$ - which can be identified with the dual bundle $\xi^{*}$ if $\xi$ is given as the kernel of a one-form, or with $\xi$ itself if $M$ is equipped with a metric. Two $\xi^{*}$-framed curves $(C, \gamma)$ and $\left(C^{\prime}, \gamma^{\prime}\right)$ are cobordant if there exists a compact surface $S$ in $[0,1] \times M$, with $\partial S=(\{0\} \times C) \cup\left(\{1\} \times C^{\prime}\right)$, such that $\gamma \cup \gamma^{\prime}:\left.\left.\nu S\right|_{\partial S} \rightarrow \xi^{*}\right|_{\partial S}$ extends to a bundle equivalence $v S \rightarrow \xi^{*} \mid S$, where indeed $\xi^{*}$ stands here for its pullback over $[0,1] \times M$ (we will consistently use the same notation for a bundle over $M$ and its pullback over $[0,1] \times M)$.

Proof From hyperplane fields to $\xi^{*}$-framed curves, the correspondence goes as follows. For a generic hyperplane field $\eta \in \mathcal{P} \mathcal{F}_{c}(M)$, the set $C(\xi, \eta)$ of points where $\xi$ coincides with $-\eta$ is a $\xi^{*}$-framed curve. In fact, if we regard $\xi$ and $\eta$ as sections of the sphere cotangent bundle $S T^{*} M$ and denote their images by $X$ and $Y$, respectively, $C=C(\xi, \eta)$ is the projection to $M$ of the intersection $X \cap(-Y)$. If $X$ and $(-Y)$ are transverse to each other, $C$ is a closed curve. Furthermore, over any point $p$ in $M$, the tangent space of $X$ determines a projection from the tangent space of $S T^{*} M$ at $\xi(p)$ to $\xi^{*}(p)$, the tangent space of the fiber $S T_{p}^{*} M$. Along the curve $C$, since transversality holds, the composition of the differential of $-\eta$ with this projection provides the required bundle equivalence $\gamma:\left.v C \rightarrow \xi^{*}\right|_{C}$. Clearly, the cobordism class of the $\xi^{*}$-framed curve $(C, \gamma)$ only depends on the homotopy class of $\eta$.

The correspondence in the other direction is a version of the Thom-Pontryagin construction. Given a $\xi^{*}$-framed curve $(C, \gamma)$, the considerations above show how $\gamma$ defines a germ $\eta_{0}$ of hyperplane field near $C$ (actually only the 1 -jet of it) that coincides 
transversally with $-\xi$ along $C$. Then pick one-forms $\alpha$ and $\beta_{0}$ defining $\xi$ and $\eta_{0}$, respectively, choose a function $\rho: M \rightarrow[0,1]$ equal to 1 in the $\varepsilon$-neighborhood of $C$ and to 0 out of the $2 \varepsilon$-neighborhood of $C$, and consider the one-form $\beta=(1-\rho) \alpha+\rho \beta-0$. For $\varepsilon$ sufficiently small, $\beta$ is defined everywhere and non-singular, and its kernel $\eta$ is a hyperplane field whose associated $\xi^{*}$-framed curve is $(C, \gamma)$. The parametric version of this construction associates a homotopy of hyperplane fields to any cobordism between $\xi^{*}$-framed curves, so the correspondence between homotopy classes and cobordism classes is well-defined and one-to-one.

Example 9 (The Pontryagin isomorphism) Let $\xi$ be a hyperplane field on $B=\mathbf{D}^{n}$. Choose a positive trivialization of $T B$ in which $\xi$ is spanned by the first $(n-1)$ basis vectors, and note that $\xi$ and $\xi^{*}$ are then also trivialized. Thus, $\xi^{*}$-framed curves in $B$ are just usual framed curves while elements of $\mathcal{P} \mathcal{F}_{c}(B)$ are identified with maps $B=\mathbf{D}^{n} \rightarrow \mathbf{S}^{n-1}$ that are constant equal to $(0, \ldots, 0,1)$ near $\partial B$. So we get a natural one-to-one correspondence

$$
\pi_{n} \mathbf{S}^{n-1}=\pi_{0} \mathcal{P} \mathcal{F}_{c}(B) \longrightarrow \Omega_{1}\left(B ; \xi^{*}\right)=\Omega_{1}\left(B ; \underline{\mathbb{R}}^{n-1}\right)
$$

which is indeed independent of $\xi$ and of the trivialization since it takes the homotopy class of any generic $g: \mathbf{D}^{n} \rightarrow \mathbf{S}^{n-1}$ to the cobordism class of the framed curve $\left(C_{g}, \gamma_{g}\right)$ defined as follows:

- $C_{g}$ is the fiber $g^{-1}(q)$ where $q=(0, \ldots, 0,-1) \in \mathbf{S}^{n-1}$ is supposed to be a regular value;

- $\gamma_{g}:\left.v C_{g} \rightarrow \xi^{*}\right|_{C_{g}}=C_{g} \times T_{-q} \mathbf{S}^{n-1}$ is given by $\gamma_{g}(p, w)=(p,-d g(p) w)$.

Furthermore, this correspondence induces a group structure on $\Omega_{1}\left(B ; \underline{\mathbb{R}}^{n-1}\right)$ : any two framed curves can be individually isotoped into disjoint balls and the sum of their cobordism classes is then the cobordism class of the union.

Now let $C \subset$ Int $B$ be a circle in the $x_{1} x_{2}$-plane and let $\gamma^{1}: \nu C \rightarrow C \times \mathbb{R}^{n-1}$ be its standard normal framing - the normal vector in the plane followed by the canonical basis of the remaining $\mathbb{R}^{n-2}$. Any loop $u: \mathbf{S}^{1}=C \rightarrow \mathrm{SO}_{n-1}$, considered as an automorphism of $C \times \mathbb{R}^{n-1}$, can be composed with $\gamma^{1}$ to give a framing $\gamma^{u}=u \cdot \gamma^{1}$. The resulting map

$$
\pi_{1} \mathrm{SO}_{n-1} \longrightarrow \Omega_{1}\left(B ; \underline{\mathbb{R}}^{n-1}\right)=\pi_{n} \mathbf{S}^{n-1}
$$

is a group homomorphism (for, if copies of $\left(C, \gamma^{u}\right)$ and $\left(C, \gamma^{v}\right)$ are placed in disjoint balls, their union is cobordant to $\left.\left(C, \gamma^{u v}\right)\right)$ and is surjective (because any cooriented closed curve in $B$ is cobordant to $C$ ). By a theorem of L Pontryagin, this map is indeed an isomorphism, and the arguments in the proof below will actually show that it is injective. 
Proof of Proposition 7 Note first that the orientations of $\xi$ and $M$ induce an orientation of $\xi^{*}$. Therefore, any $\xi^{*}$-framed curve $(C, \gamma)$ is (co) oreiented by $\gamma:\left.\nu C \rightarrow \xi^{*}\right|_{C}$, and so it determines a 1 -cycle whose homology class depends only on the cobordism class of $(C, \gamma)$. This gives a map $\Omega_{1}\left(M ; \xi^{*}\right) \rightarrow H_{1}(M ; \mathbb{Z})$ whose composition with the bijection $\pi_{0} \mathcal{P} \mathcal{F}_{c}(M) \rightarrow \Omega_{1}\left(M ; \xi^{*}\right)$ sends the homotopy class of a hyperplane field $\eta$ to the obstruction class $c(\xi, \eta)$. The cocycle $c$ is then surjective for, in dimension $n \geq 2$, any homology class in $H_{1}(M ; \mathbb{Z})$ can be represented by an embedded, oriented, closed curve, and any such curve $C$ admits a $\xi^{*}$-framing since both $\nu C$ and $\left.\xi^{*}\right|_{C}$ are trivial bundles.

Consider two hyperplane fields $\xi, \eta \in \mathcal{P} \mathcal{F}_{c}(M)$. By definition, their homotopy classes lie in the same $\pi_{n} \mathbf{S}^{n-1}$-orbit if and only if $\eta$ is homotopic to a hyperplane field that coincides with $\xi$ out of a ball, and this property clearly implies $c(\xi, \eta)=0$. Conversely, if $c(\xi, \eta)$ is zero and if $\xi$ and $\eta$ are generic, the curve $C=C(\xi, \eta)$ consisting of points where $\xi=-\eta$ is nullhomologous. Hence, $C$ is cobordant to $C^{\prime}=\partial D$ where $D$ is an embedded disk disjoint from $C$. Let $S \subset[0,1] \times M$ be a connected, oriented, compact surface with $\partial S=(\{0\} \times C) \cup\left(\{1\} \times C^{\prime}\right)$. Since $S$ retracts onto the union of $\{0\} \times C$ and a graph, the $\xi^{*}$-framing of $C$ extends over $S$. Therefore, by Lemma $8, \eta$ is homotopic to a hyperplane field $\eta^{\prime}$ such that $C\left(\xi, \eta^{\prime}\right)=C^{\prime}$, and using an affine homotopy away from $C^{\prime}$, we can indeed make $\eta^{\prime}$ equal to $\xi$ out of a small neighborhood of $C^{\prime}=\partial D$. Thus, the homotopy classes of $\xi$ and $\eta$ are in the same orbit under $\pi_{n} \mathbf{S}^{n-1}$.

It remains to compute the stabilizer of the homotopy class of $\xi$. To do this, take a ball $B \subset M$ parameterized by $\mathbf{D}^{n}$. As explained in Example 9, the cobordism classes corresponding via Lemma 8 to the $\pi_{n} \mathbf{S}^{n-1}$-orbit of the homotopy class of $\xi$ are represented by the $\xi^{*}$-framed curves $\left(C, \gamma^{u}\right)$, where $C$ is a fixed circle in $B$ and $u$ a loop in $\mathrm{SO}_{n-1}$. Our task is to determine the loop classes $[u] \in \pi_{1} \mathrm{SO}_{n-1}$ for which the $\xi^{*}$-framing $\gamma^{u}$ extends over some connected, oriented, compact surface in $[0,1] \times M$ bounded by $\{0\} \times C$.

Let $S$ be such a surface and choose a trivialization of the bundle $v S$ - and thereby also a trivialization of $\nu C$. This choice identifies bundle equivalences $v S \rightarrow \xi^{*} \mid S$ with trivializations of $\xi^{*} \mid S$. But any two trivializations of this bundle are homotopic over the boundary because they differ by a map $S \rightarrow \mathrm{SO}_{n-1}$, and the restriction of such a map to $\partial S=\{0\} \times C$ (which is connected) is nullhomotopic. This shows that, up to homotopy, there exists a unique $\xi^{*}$-framing of $C$ that extends over the given surface $S$.

Consider now a disk $D \subset[-1,0] \times M$ bounded by $\{0\} \times C$ and whose projection to $M$ is the affine disk spanned by $C$ in $B$. The (unique) $\xi^{*}$-framing of $C$ that 
extends to $D$ is the standard framing $\gamma^{1}$ (see Example 9). Denote by $\bar{S} \subset \mathbb{R} \times M$ the closed, connected, oriented surface obtained by smoothing $S \cup(-D)$ in the obvious way. Since $T(\mathbb{R} \times M)=T M \oplus \underline{\mathbb{R}}=\xi \oplus \underline{\mathbb{R}}^{2}$ while $T(\mathbb{R} \times M) \mid \bar{S}=\nu \bar{S} \oplus T \bar{S}-$ and $T \bar{S} \oplus \underline{\mathbb{R}}=\underline{\mathbb{R}}^{3}$, the bundles $\xi^{*} \mid \bar{S}$ and $\nu \bar{S}$ are stably equivalent. If $n \geq 4$ then $\xi^{*} \mid \bar{S}$ and $v \bar{S}$ are indeed equivalent (because a vector bundle of rank at least four over a surface has a connected space of non-vanishing sections), and so the $\xi^{*}$-framing of $C$ that extends over $D$ also extends over $S$. Therefore, $\gamma^{1}$ is the unique $\xi^{*}$-framing of $C$ that is nullcobordant, and so $\pi_{n} \mathbf{S}^{n-1}=\mathbb{Z} / 2 \mathbb{Z}$ acts freely. If $n=3$, however, the bundle $v \bar{S}$ is trivial but $\xi^{*} \mid \bar{S}$ is not in general: given trivializations of $\left.\xi^{*}\right|_{D}$ and $\left.\xi^{*}\right|_{S}$, the induced trivializations of $\left.\xi^{*}\right|_{C}$ differ by a map $C \rightarrow \mathrm{SO}_{2}$ whose degree is the Euler class $e(\xi)$ of $\xi$ evaluated on $[\bar{S}] \in H_{2}(M ; \mathbb{Z})$. Thus, the $\xi^{*}$-framing $\gamma^{u}$ of $C$ extends over $S$ if and only if $\langle e(\xi),[\bar{S}]\rangle=[u] \in \pi_{1} \mathrm{SO}_{2}$. This completes the proof.

Let's now return to our three-dimensional framework. It follows from Proposition 7 that the stabilizer of the homotopy class of a plane field $\xi$ under the action of $\pi_{3} S^{2}=\mathbb{Z}$ is $|\xi| \mathbb{Z}$, where $|\xi|$ denotes the divisibility of the (torsion-free part of the) Euler class of $\xi$. For any plane field $\eta$ homologous to $\xi$, we will call relative framing of $\xi$ and $\eta$ the element $d(\xi, \eta) \in\{0, \ldots,|\xi|-1\}$ which takes the homotopy class of $\xi$ to that of $\eta$. As an illustration, we can recover a calculation of W Neumann and L Rudolph:

Lemma 10 (Neumann-Rudolph [10]) Let $\xi$ denote the standard contact structure on $\mathbf{S}^{3}$ - that is, the plane field orthogonal to the Hopf fibers - and $\xi^{-}$the plane field associated with the negative Hopf link $H^{-}$. Then the relative framing $d\left(\xi, \xi^{-}\right)$is equal to 1 .

Proof Since the Hopf flow preserves the open book given by $\mathrm{H}^{-}$(whose mapping to the circle is the argument of the Hopf fibration: see Example 6), we can arrange that it preserves $\xi^{-}$too (just construct $\xi^{-}$as indicated in the introduction). On the other hand, $\xi$ is the plane field orthogonal to the Hopf fibers, and is also invariant under the Hopf flow. Therefore, the $\xi^{*}$-framed curve $(C, \gamma)$ determined by $\xi^{-}$is also invariant. Clearly, the curve $C$ (the set of points where $\xi^{-}$coincides with $-\xi$ ) is the component $\left\{r_{2}=0\right\}$ of $H^{-}$, which is transverse to $\xi$. Then, if we identify $\xi^{*}$ with $\xi$ using the metric of $\mathbf{S}^{3}$, the bundle equivalence $\gamma:\left.\nu C \rightarrow \xi\right|_{C}=\nu C$ is (homotopic to) the identity.

Now let $D$ be a disk bounded by $C$ in $[0,1] \times \mathbf{S}^{3}$. The trivialization of $\nu C$ that extends to $v D$ differs from the trivialization of $\xi \mid C$ that extends to $\xi \mid D$ by one twist: indeed, $\xi$ admits a global non-vanishing section and the linking number of $C$ and its push-off along this section is equal to -1 . This proves that the relative framing $d\left(\xi, \xi^{-}\right)$is equal to 1 . 


\section{Contact structures}

We briefly present here some notions and results of three-dimensional contact geometry that we will invoke to prove Theorem 1. A contact form on an oriented three-manifold $M$ is a one-form $\alpha$ whose exterior product with $d \alpha$ is everywhere positive - with respect to the orientation of $M$. A contact structure on $M$ is a (co) oriented plane field $\xi$ which is the kernel of some contact form, and a contact manifold is a manifold equipped with a contact structure. A fundamental property of contact structures, established by J Gray, is that they are $\mathcal{C}^{1}$-stable: if $\xi_{s}, s \in[0,1]$, is a path of contact structures on a closed manifold $M$, then there exists an isotopy $\phi_{S}$ of $M$ such that $\phi_{0}=\mathrm{id}$ and $\phi_{s *} \xi_{0}=\xi_{s}$ for all $s \in[0,1]$. Thus, two contact structures on a closed manifold $M$ are isotopic if and only if they are in the same homotopy class of contact structures (that is, the same connected component of the space of contact structures).

The possibility of constructing contact structures on three-manifolds from open books was discovered by Thurston and Winkelnkemper [12]. However, the systematic study of the relations between these two geometric objects is much more recent (see the article [6] by the first author) and is based on the following:

Definition 11 Let $M$ be a closed oriented three-manifold. We say that a contact structure $\xi$ on $M$ is carried by an open book $(K, \theta)$ if it is the kernel of a one-form $\alpha$ satisfying the following conditions:

- $\alpha$ induces a positive non-singular form on $K$;

- $d \alpha$ induces a positive area form on each fiber of $\theta$.

Any such one-form $\alpha$ is said to be adapted to $(K, \theta)$.

With this terminology, the main result of Thurston and Winkelnkemper [12] is that the set of contact structures carried by a given open book is non-empty. It is easy to check that this set is also open and contractible in the space of all contact structures on $M$ (see the proof of Lemma 17). In particular, according to Gray's stability theorem, all the contact structures it contains belong to the same isotopy class. For this reason, we often speak of "the contact structure associated with the open book", this contact structure being defined only up to isotopy.

Remark 12 The contact structure associated with an open book $(K, \theta)$ - in a closed oriented three-manifold $M$ - belongs to the homotopy class of plane fields associated with $(K, \theta)$. In fact, if $\alpha_{0}$ is a one-form defining the plane field associated with $(K, \theta)$ 
as in the introduction (with $N$ small enough) and if $\alpha_{1}$ is a contact form adapted to $(K, \theta)$, then all forms $(1-t) \alpha_{0}+t \alpha_{1}, t \in[0,1]$, are non-singular, and so their kernels yield the desired homotopy of plane fields.

Example 13 The standard contact structure $\xi$ on $\mathbf{S}^{3} \subset \mathbb{C}^{2}$ is defined by the oneform $\alpha=r_{1}^{2} d \theta_{1}+r_{2}^{2} d \theta_{2}$. This contact form is adapted to the trivial open book $\left(U, \theta_{1}\right)$ for it induces on each fiber of $\theta_{1}$ the one-form $r_{2}^{2} d \theta_{2}$. It is also adapted to the open book $\left(H^{+}, \theta_{1}+\theta_{2}\right)$, for it induces on each fiber of $\theta_{1}+\theta_{2}$ the one-form $\left(2 r_{1}^{2}-1\right) d \theta_{1}=\left(2 r_{2}^{2}-1\right) d \theta_{2}$.

The above example shows that a given contact structure may be carried by several open books. Indeed, according to a previous article by the first author [6], any contact structure is carried by many open books but we have the following stable equivalence theorem:

Theorem 14 (Giroux [6]) On a closed oriented three-manifold, two open books carrying the same contact structure admit isotopic positive stabilizations.

The last ingredient of contact geometry we will need is the classification of overtwisted contact structures, due to Y Eliashberg. A contact structure $\xi$ on a three-manifold $M$ is overtwisted if there exists a simple closed curve $L \subset M$ with the following properties:

- $L$ is Legendrian, that is, is tangent to $\xi$ at each point;

- $L$ is unknotted, that is, bounds a disk;

- the Thurston-Bennequin number of $L-$ that is, the linking number of $L$ and its push-off along the normal vector to $\xi-$ is non-negative.

Overtwisted contact structures have an extremely simple classification:

Theorem 15 (Eliashberg [3]) On a closed oriented three-manifold, two overtwisted contact structures are isotopic if and only if they are in the same homotopy class of plane fields.

\section{Proof of the stable equivalence theorem}

Let $(K, \theta)$ be an open book in a closed oriented three-manifold $M$, with associated plane field $\xi$, and let $\left(K^{+}, \theta^{+}\right)$and $\left(K^{-}, \theta^{-}\right)$denote open books obtained from $(K, \theta)$ by $H^{+}$-plumbing and $H^{-}$-plumbing, respectively. 
Lemma 16 The plane field $\xi^{+}$associated with $\left(K^{+}, \theta^{+}\right)$is homotopic to $\xi$ while the plane field $\xi^{-}$associated with $\left(K^{-}, \theta^{-}\right)$is homologous to $\xi$ with relative framing $d\left(\xi, \xi^{-}\right)$equal to 1 .

Proof Consider the open books in $\mathbf{S}^{3}$ given by $H^{+}$and by the unknot $U$. After isotoping one of them, we may assume that they have a common plumbing ball $B$ (see Section 1) in which they coincide as well as their associated plane fields. Now these plane fields are homotopic to each other (according to Example 13 and Remark 12, both are homotopic to the standard contact structure) and, since $\mathbf{S}^{2}$ is simply connected, they are also homotopic relative to $B$. Therefore, $\xi^{+}$is homotopic to the plane field associated with the open book obtained by plumbing $(K, \theta)$ with $U$. But this open book is isotopic to $(K, \theta)$, so $\xi^{+}$is homotopic to $\xi$. On the other hand, $\xi^{-}$and $\xi$ are homologous since they coincide out of a ball. Next, arguing as above, we see that the relative framing $d\left(\xi, \xi^{-}\right)$is equal to the relative framing in $\mathbf{S}^{3}$ of the standard contact structure and the plane field associated with the negative Hopf link $H^{-}$. Then the result follows from Lemma 10.

The next lemma is essentially due to Torisu:

Lemma 17 (Torisu [13]) The contact structure $\xi^{-}$associated with $\left(K^{-}, \theta^{-}\right)$is overtwisted.

Proof Let's say that a one-form $\beta$ on a compact oriented surface is admissible if it induces a positive non-singular form on the boundary and if its differential $d \beta$ is a positive area form in the interior. A contact form adapted to an open book clearly induces an admissible one-form on each page. The existence and uniqueness - up to isotopy - of contact structures carried by an open book is mostly due to the contractibility of the space of admissible forms on a given surface. This contractibility also allows to construct an adapted contact form inducing a prescribed admissible form on a given page (Thurston-Winkelnkemper [12]).

Now let $F^{-}=F \cup A^{-}$be a page of $\left(K^{-}, \theta^{-}\right)$, where $F$ is a page of $(K, \theta)$ and $A^{-}$ a negative Hopf band. Since the core curve $L$ of $A^{-}$is homologically non-zero in $F^{-}$, there exists an admissible form $\beta^{-}$that vanishes at each point of $L$. Then consider a contact form $\alpha^{-}$on $M$ which is adapted to $\left(K^{-}, \theta^{-}\right)$and induces $\beta^{-}$ on $F^{-}$. For the contact structure $\xi^{-}$defined by $\alpha^{-}$, the curve $L$ is Legendrian and unknotted. Moreover, the normal vector to $\xi^{-}$along $L$ is the normal vector to $A^{-}$, so the Thurston-Bennequin number of $L$ is equal to 1 . Thus, the contact structure $\xi^{-}$is overtwisted. 
Proof of Theorem 1 If two open books admit isotopic stabilizations, their associated plane fields are homologous since the homology class does not change under $H^{ \pm}$ plumbing. Suppose now that $(K, \theta)$ and $\left(K^{\prime}, \theta^{\prime}\right)$ are two open books in $M$ whose associated contact structures $\xi$ and $\xi^{\prime}$ are homologous as plane fields. Let $d$ denote the relative framing $d\left(\xi, \xi^{\prime}\right)$ and consider an open book $\left(K^{\prime \prime}, \theta^{\prime \prime}\right)$ obtained from $(K, \theta)$ by $d$ successive $H^{-}$-plumbings. According to Lemma 16 and Lemma 17, the contact structure $\xi^{\prime \prime}$ associated with $\left(K^{\prime \prime}, \theta^{\prime \prime}\right)$ is in the same homotopy class of plane fields as $\xi^{\prime}$ and is overtwisted provided $d \geq 1$. Applying one more $H^{-}$-plumbing to both $\left(K^{\prime}, \theta^{\prime}\right)$ and $\left(K^{\prime \prime}, \theta^{\prime \prime}\right)$ if necessary, we may assume that $\xi^{\prime}$ and $\xi^{\prime \prime}$ are both overtwisted. Then it follows from Eliashberg's Theorem 15 that $\xi^{\prime}$ and $\xi^{\prime \prime}$ are actually isotopic. Hence Theorem 14 implies that $\left(K^{\prime}, \theta^{\prime}\right)$ and $\left(K^{\prime \prime}, \theta^{\prime \prime}\right)$ admit isotopic positive stabilizations, so $(K, \theta)$ and $\left(K^{\prime}, \theta^{\prime}\right)$ admit isotopic stabilizations.

Remark 18 The above proof shows that, while there is no control on the number of necessary $\mathrm{H}^{+}$-plumbings, the number of necessary $\mathrm{H}^{-}$-plumbings can be bounded a priori in terms of the relative framing of $\xi$ and $\xi^{\prime}$, namely, by

$$
2+\min \left\{d\left(\xi, \xi^{\prime}\right), d\left(\xi^{\prime}, \xi\right)\right\}
$$

Corollaries 2 and 3 follow readily from Theorem 1. Corollary 4 follows similarly from the following refined version of Theorem 1 :

Theorem 19 In a closed oriented three-manifold, two open books with connected bindings and homologous associated plane fields admit isotopic stabilizations which can be obtained by finitely many successive $T^{+}$-plumbings and $E$-plumbings.

Proof Suppose that $(K, \theta)$ and $\left(K^{\prime}, \theta^{\prime}\right)$ are two open books in $M$ whose binding are connected and whose associated contact structures $\xi$ and $\xi^{\prime}$ are homologous as plane fields. Since $E$-plumbing is a composition of an $H^{+}-$and an $H^{-}$-plumbing, its effect on the homotopy class and the isotopy type of the associated contact structure is the same as the effect of $H^{-}$-plumbing. Therefore, after performing a number of $E$-plumbings on our open books (see the proof of Theorem 1, we may assume that $\xi$ and $\xi^{\prime}$ are overtwisted and homotopic as plane fields, and hence isotopic by Eliashberg's Theorem 15. Then we conclude with the following refined version of Theorem 14: on a closed oriented three-manifold, two open books carrying the same contact structure and having connected bindings admit isotopic positive stabilizations which can be obtained by $T^{+}$-plumbings.

Proof of Corollary 5 Corollary 3 shows that the Grothendieck group $\Gamma$ of fibered links in $\mathbf{S}^{3}$ is generated by the Hopf links $H^{+}$and $H^{-}$. To complete the proof, we 
proceed as Neumann-Rudolph in [10]. To each fibered link $K$ in $\mathbf{S}^{3}$ we assign two integers, $\mu(K)$ and $\lambda(K)$ :

- $\mu(K)$ is the Milnor number of $K$, that is, the first Betti number of a fiber Seifert surface (a page of the corresponding open book);

- $\lambda(K)$ is the "enhanced Milnor number", that is, relative framing of the plane field associated with the unknot (the standard contact structure for instance) and the plane field associated with $K$.

The additivity of $\mu$ and $\lambda$ under plumbing (which follows from our discussion in Sections 1 and 2 - see also the proof of Lemma 16) implies that the pair $(\mu, \lambda)$ induces a homomorphism from $\Gamma$ to $\mathbb{Z}^{2}$. By Lemma 10 this homomorphism maps the generators $H^{+}$and $H^{-}$to $(1,0)$ and $(1,1)$, respectively, and so it is an isomorphism.

The calculation of the Grothendieck group of fibered knots is analogous.

\section{References}

[1] J Cerf, Sur les difféomorphismes de la sphère de dimension trois $\left(\Gamma_{4}=0\right)$, Lecture Notes in Math. 53, Springer, Berlin (1968) MR0229250

[2] E Dufraine, Classes d'homotopie de champs de vecteurs Morse-Smale sans singularité sur les fibrés de Seifert, Enseign. Math. (2) 51 (2005) 3-30 MR2154619

[3] Y Eliashberg, Classification of overtwisted contact structures on 3-manifolds, Invent. Math. 98 (1989) 623-637 MR1022310

[4] D Gabai, The Murasugi sum is a natural geometric operation, from: "Low-dimensional topology (San Francisco, Calif., 1981)", Contemp. Math. 20, Amer. Math. Soc., Providence, RI (1983) 131-143 MR718138

[5] D Gabai, The Murasugi sum is a natural geometric operation. II, from: "Combinatorial methods in topology and algebraic geometry (Rochester, N.Y., 1982)", Contemp. Math. 44, Amer. Math. Soc., Providence, RI (1985) 93-100 MR813105

[6] E Giroux, Géométrie de contact: de la dimension trois vers les dimensions supérieures, from: "Proceedings of the International Congress of Mathematicians, Vol. II (Beijing, 2002)", Higher Ed. Press, Beijing (2002) 405-414 MR1957051

[7] R E Gompf, Handlebody construction of Stein surfaces, Ann. of Math. (2) 148 (1998) 619-693 MR1668563

[8] J Harer, How to construct all fibered knots and links, Topology 21 (1982) 263-280 MR649758

[9] F Laudenbach, S Blank, Isotopie de formes fermées en dimension trois, Invent. Math. 54 (1979) 103-177 MR550181 
[10] W D Neumann, L Rudolph, The enhanced Milnor number in higher dimensions, from: "Differential topology (Siegen, 1987)", Lecture Notes in Math. 1350, Springer, Berlin (1988) 109-121 MR979336

[11] J R Stallings, Constructions of fibred knots and links, from: "Algebraic and geometric topology (Proc. Sympos. Pure Math., Stanford, Calif. 1976), Part 2”, Proc. Sympos. Pure Math. XXXII, Amer. Math. Soc., Providence, R.I. (1978) 55-60 MR520522

[12] W P Thurston, HE Winkelnkemper, On the existence of contact forms, Proc. Amer. Math. Soc. 52 (1975) 345-347 MR0375366

[13] I Torisu, Convex contact structures and fibered links in 3-manifolds, Internat. Math. Res. Notices (2000) 441-454 MR1756943

[14] V G Turaev, Euler structures, nonsingular vector fields, and Reidemeister-type torsions, Izv. Akad. Nauk SSSR Ser. Mat. 53 (1989) 607-643, 672 MR1013714

[15] F Waldhausen, On irreducible 3-manifolds which are sufficiently large, Ann. of Math. (2) 87 (1968) 56-88 MR0224099

École Normale Supérieure de Lyon, 69364 Lyon cedex 07, France

Massachusetts Institute of Technology, Cambridge, Massachusetts 02139, USA

Emmanuel.Giroux@ens-lyon.fr, ndg@mit.edu

Proposed: Rob Kirby

Seconded: David Gabai, Peter Ozsváth
Received: 19 September 2005

Accepted: 28 October 2005 